\title{
Comment on "Acute Kidney Injury in COVID-19 Patients: An Inner City Hospital Experience and Policy Implications"
}

\author{
Nick Flynn \\ Clinical Biochemistry, Cambridge University Hospitals NHS Foundation Trust, Addenbrooke's Hospital, \\ Cambridge Biomedical Campus, Cambridge, UK
}

In a recent article in American Journal of Nephrology [1], Zahid et al. [1] refer to "creatinine kinase" (sic) in the article abstract. Rather than "creatinine kinase," presumably the authors were instead referring to the enzyme creatine kinase. Creatine kinase (also known as creatine phosphokinase) catalyses the reversible phosphorylation of creatine to phosphocreatine, is frequently measured as a marker of muscle damage, and is commonly abbreviated as "CK" [2]. Creatinine is neither a product nor substrate for creatine kinase and is instead formed from creatine and phosphocreatine via non-enzymatic reactions.

The mistake of referring to creatine kinase as creatinine kinase is common in the medical literature. It is like- ly that most readers understood the authors as they intended. However, this misspelling has the potential to cause confusion, can complicate literature searches, and may result in a loss of reader confidence in what might otherwise be a high-quality publication.

\section{References}

1 Zahid U, Ramachandran P, Spitalewitz S, Alasadi L, Chakraborti A, Azhar M, et al. Acute kidney injury in COVID-19 patients: an inner city hospital experience and policy implications. Am J Nephrol. 2020;51(10): 786-96.

2 Wyss M, Kaddurah-Daouk R. Creatine and creatinine metabolism. Physiol Rev. 2000 Jul;80(3):1107-213. 